\title{
Static and Dynamic Aeroelastic Tailoring with Variable Camber Control
}

\author{
Bret K. Stanford ${ }^{1}$ \\ NASA Langley Research Center, Hampton, VA, 23681
}

\begin{abstract}
This paper examines the use of a Variable Camber Continuous Trailing Edge Flap (VCCTEF) system for aeroservoelastic optimization of a transport wingbox. The quasisteady and unsteady motions of the flap system are utilized as design variables, along with patch-level structural variables, towards minimizing wingbox weight via maneuver load alleviation and active flutter suppression. The resulting system is, in general, very successful at removing structural weight in a feasible manner. Limitations to this success are imposed by including load cases where the VCCTEF system is not active (open-loop) in the optimization process, and also by including actuator operating cost constraints.
\end{abstract}

\section{Introduction}

Distributed control surfaces along the trailing-edge of a wing structure can have a substantial impact upon the aeroelastic stiffness, mass, and damping properties of that structure [1]. The quasi-steady and unsteady deflection patterns/history of these control surfaces may be optimized during an aeroelastic tailoring procedure, alongside more traditional design variables such as structural thicknesses of the skins, spars, ribs, and stringers. Designing both sets of design variables at once allows the optimizer to take full advantage of the strong synergies that exist between the two. Quasi-steady flap deflections may be optimized for maneuver load alleviation and cruise drag (or fuel burn) reduction [2][3]. Unsteady flap deflections (where each control surface oscillates about its mean quasi-steady position) may be used for active flutter control, gust alleviation, ride quality control, etc. [4]-[7].

Despite the efficacy of control surface rotations for aeroelastic optimization, two important considerations should be included in the process, in the form of design constraints. The first is rooted in certification concerns: will the aeroelastic response of the wing be sufficiently safe in the unlikely event that the control surfaces are inactive? This risk may be mitigated by increasing the level of importance of open-loop load cases (where control surfaces are not actuated/included) relative to closed-loop cases (where they are) during the optimization process. As is often the case with aerospace systems [8], there is a strong trade-off between this form of risk reduction and overall structural performance (weight, e.g.). Design constraints attached to open-loop cases will dilute the effectiveness of the control surface actuation design variables.

A second consideration may be the cost to actuate the control surfaces, measured in terms of the work done by applied hinge moments, or controller cost metrics. Distributed control surfaces are adept at maneuver load alleviation, for example, but maintaining large deflections under strong aerodynamic loading may increase the overall operating costs of the airplane. As above, one may expect a trade-off between these actuation cost metrics and the aeroelastic performance of the control surfaces. In addition to actuation cost metrics, constraints which recognize actuation limitations must also be enforced: maximum hinge moments, deflection limits, etc.

Trade-offs between performance, open-loop risk reduction, and actuator cost have been studied in aeroelastic and aeroservoelastic optimization settings before [9][10]. The focus of the current work is to explore these issues for a subsonic transport configuration outfitted with Variable Camber Continuous Trailing Edge Flaps: VCCTEF [11]. This concept involves segmented flaps distributed along the trailing-edge, connected to one another with an elastomeric sheet, to maintain a spatially-continuous surface. Each discrete flap is composed of 3 chordwise segments: the first two (upstream) are actuated in a quasi-steady manner with shape memory alloy (SMA) torquetube actuators, while the third segment along the trailing-edge is actuated dynamically with an electric drive motor. This actuation concept is conducted on the un-deflected Common Research Model (uCRM) [12], a jig shape of the $1 \mathrm{~g}$ wing shape model developed in Ref. [13]. The uCRM is a generic transport configuration with a standard ribspar wingbox.

The work demonstrated here is conducted in two stages. First, VCCTEF is utilized for quasi-steady maneuver load alleviation. Patch-wise structural design variables and flap actuation design variables are used to minimize the weight of the wingbox, subject to stress, buckling, and hinge moment constraints. Both open- and closed-loop maneuver loads are considered, but flap actuation is removed from the open-loop loads. The required safety factor

\footnotetext{
${ }^{1}$ Research Aerospace Engineer, Aeroelasticity Branch, bret.k.stanford@nasa.gov, AIAA Senior Member.
} 
is less for these open-loop loads (diminishing their importance relative to the closed-loop loads), allowing for mass reduction, albeit with greater risk. Secondly, open- and closed-loop flutter constraints are added to the aeroelastic optimization problem. Similar to the quasi-steady problem, the open-loop case does not benefit from flap actuation, but the required flutter margin is less as well. This allows for overall reductions in structural weight, subject to potential limitations in actuation cost.

\section{Transport Configuration Test Case}

All of the work in this paper is conducted on the conceptual Common Research Model (CRM). The model presented in Ref. [13] is a $1 \mathrm{~g}$ flying shape suitable for rigid aerodynamic analysis. An un-deflected jig shape version (uCRM) developed in Ref. [12] is suitable for aeroelastic analysis, and is used here. This transonic transport configuration has a wing span of $58.7 \mathrm{~m}$, a mean aerodynamic chord of $7.0 \mathrm{~m}$, an aspect ratio of 9 , a taper ratio of 0.275 , a sweep angle of $35^{\circ}$, and a cruise Mach number of 0.85 .

The topology of the wingbox developed in Ref. [12] is also used here, seen in Figure 1. This structure consists of an upper surface skin, a lower surface skin, a leading-edge spar (located at $10 \%$ chord at the root and $35 \%$ at the tip), a trailing-edge spar ( $60 \%$ at both the root and the tip), and 43 ribs oriented perpendicular to the leading-edge. A carry-through structure is assumed to lie within the fuselage. All shell members are outfitted with blade stiffeners, where the pitch is equal to $21.2 \mathrm{~cm}, 30 \mathrm{~cm}, 22.5 \mathrm{~cm}$, and $15 \mathrm{~cm}$, for the upper surface skins, lower surface skins, ribs, and spars respectively. Parallel run-out stiffeners are utilized down the span, so far fewer stiffeners are found for panels at the tip than the root. The stiffeners are not modeled explicitly, but instead smeared into the shell stiffness properties [14]. T-shaped stiffeners are utilized, where the flange is bonded to the shell members. The thickness of the flange and the web are equal for all cases, as is the width of the flange and the height of the web.

The entire wing structure is constructed of aluminum ( $\left.E=73 \mathrm{GPa}, v=0.33, \rho=2780 \mathrm{~kg} / \mathrm{m}^{3}, \sigma_{Y}=420 \mathrm{MPa}\right)$, and discretized into 21,000 triangular finite elements. All nodes along the centerline are fully clamped, and all nodes along the wing root (side-of-body) are pinned.

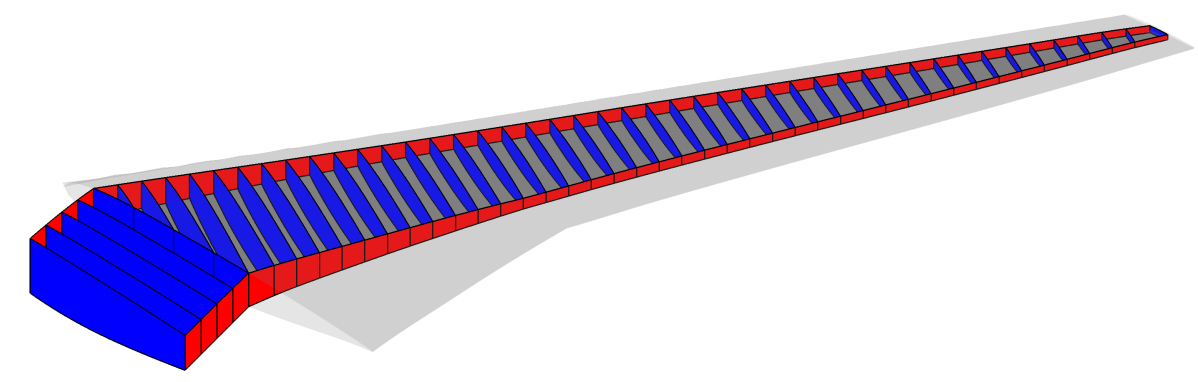

Figure 1. uCRM wingbox topology and outer mold line, taken from Ref. [12].

Twenty segmented control surfaces are distributed along the trailing-edge of the wing from root to tip, and each control surface is divided into three chordwise segments, as shown in Figure 2. All hinges are modeled with torsional spring elements, and all control surface segments are modeled as rigid linkages. The first row (upstream) of torsional springs connects the first row of VCCTEF segments to the wingbox (via interpolation elements), and is meant to emulate an SMA actuator. Lumped actuator mass is obtained by assigning worst-case aerodynamic hinge moments [15] to each torsional spring, and then assuming a proportional relationship between this hinge moment and the SMA actuator mass $(830 \mathrm{~N} \cdot \mathrm{m} / \mathrm{kg})$. Spring constants are obtained by assuming a rotational deviation of $0.2^{\circ}$ when this peak hinge moment is applied to the actuator.

A second row of torsional springs connects the first two rows of control surface segments, and these SMA actuators are sized in the same way as above. The final row of torsional springs (connecting the last two rows of control segments) models electrical rotary actuators, which are capable of much higher actuation rates than their upstream SMA counterparts, but much lower hinge moments as well for a given mass $(37 \mathrm{~N} \cdot \mathrm{m} / \mathrm{kg})$. The spring constants for the electric drive motors are sized such that every control surface wedge along this third row has the same natural vibration frequency: $20 \mathrm{~Hz}$. This is done to simplify the aeroservoelastic analyses discussed below.

The non-actuator mass of each control surface wedge is modeled with a lumped mass at its center of gravity, and is computed based on the surface area of the wedge $\left(17 \mathrm{~kg} / \mathrm{m}^{2}\right)$. Spanwise-adjacent control surface wedges are connected with elastomeric sheets along the upper and lower surface skins, to maintain a continuous surface. These sheets are modeled with planar triangular membrane finite elements, with translational but no rotational degrees of freedom. 
The structure within the leading-edge of the wing is not explicitly modeled, though an inertial effect is captured with a series of lumped masses attached to the wingbox via interpolation elements. These masses, along with similar representations for the engine and the fuel, are also shown in Figure 2. In addition to the inertial loads from the lumped masses, a thrust force is applied to the central engine node.

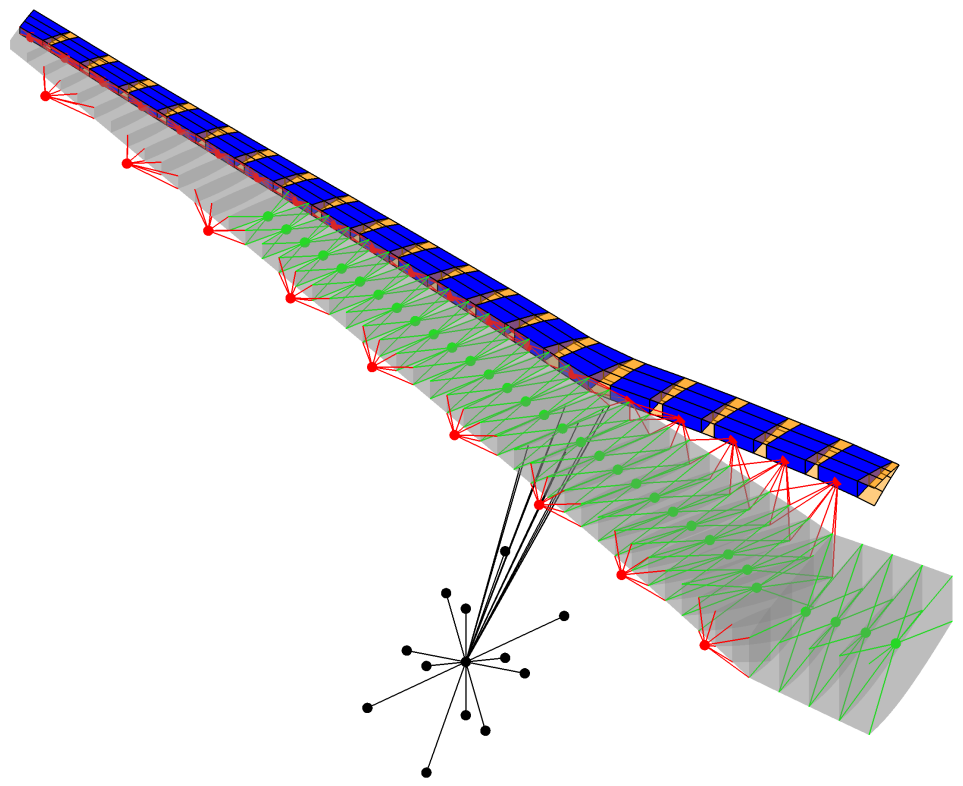

Figure 2. VCCTEF control surfaces attached to the uCRM model, and lumped mass representation of engine and fuel loading.

Aerodynamic paneling for the wing, horizontal tail, vertical tail, fuselage, and engine (the latter two represented as cruciforms) is shown in Figure 3, with a total of 5,000 panels. For static aeroelastic trim analysis, the entire vehicle representation of Figure 3 is utilized. For dynamic flutter analysis, only the wing panels are utilized. A finer aerodynamic paneling is utilized over the control surface portion of the wing (particularly towards the hinge line), in order to improve the accuracy of the aeroservoelastic loads generated by these deflections. It is finally noted that linear aerodynamic panel methods utilized in the transonic regime are only valid to first order, and will miss many potentially important effects related to shock growth and movement.

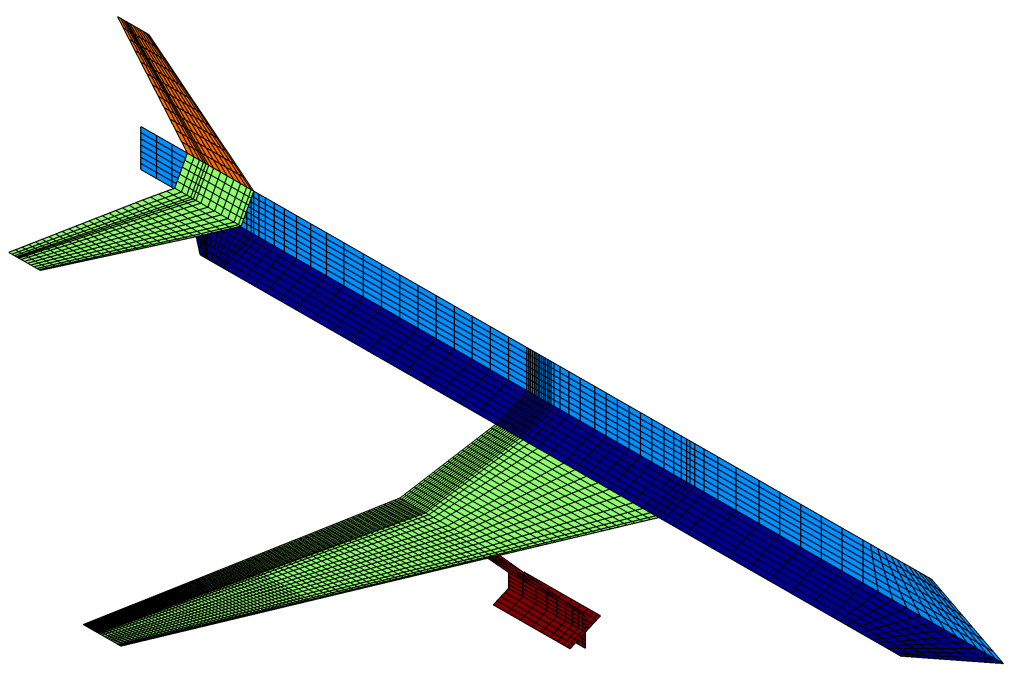

Figure 3. Aerodynamic paneling of the uCRM. 


\section{A. Trim Loads}

\section{Static Aeroelastic Modeling}

The shell finite elements used to model the wing structure are defined by a combination of linear strain triangles (LST) and discrete Kirchhoff triangles (DKT) [16]. For static airloads, a linear vortex lattice method [17] is used to model the aerodynamic lifting surfaces. A finite plate spline (FPS) method [18] is used to transfer downwash and pressures between the aerodynamic and structural/control surface modules. Only information pertaining to the wing is transferred in this way: the remaining aerodynamic surfaces are not explicitly tied to any structure.

The wingbox structure is sized across two different types of static maneuvers. The first type is an aeroelastic longitudinal maneuver (pull-up, push-over), where the system is trimmed via the angle of attack, $\alpha$, and the elevator deflection, $\delta$. The trimmed values of these two quantities are automatically found by augmenting the aero-structural coupling equations. The final equation for a longitudinal maneuver is written as:

$$
\left[\begin{array}{cccc}
\boldsymbol{K} & -q \cdot \boldsymbol{Q} & \mathbf{0} & \mathbf{0} \\
-\boldsymbol{P} & \boldsymbol{D} & -\boldsymbol{L}_{\alpha} & -\boldsymbol{L}_{\delta} \\
\mathbf{0} & q \cdot \boldsymbol{S}_{L}^{T} & 0 & 0 \\
\mathbf{0} & q \cdot \boldsymbol{S}_{m}^{T} & 0 & 0
\end{array}\right] \cdot\left\{\begin{array}{c}
\boldsymbol{x} \\
\boldsymbol{C}_{p} \\
\alpha \\
\delta
\end{array}\right\}=\left\{\begin{array}{c}
N \cdot \boldsymbol{F}_{\text {grav }}+\boldsymbol{F}_{\text {thrust }} \\
\boldsymbol{L}_{j i g} \\
N \cdot W \\
0
\end{array}\right\}+\left\{\begin{array}{c}
\boldsymbol{Q}_{H M} \\
\mathbf{0} \\
0 \\
0
\end{array}\right\} \cdot \boldsymbol{\theta}_{i n}
$$

The first row of Eq. 1 is the finite element analysis, where $\boldsymbol{K}$ is the stiffness matrix. The solution vector, $\boldsymbol{x}$, has six degrees of freedom per finite element node (three displacements and three rotations), in addition to 60 control surface spring rotations: 20 control surfaces with 3 chordwise segments each.

Forcing functions include self-weight inertial loading, $\boldsymbol{F}_{\text {grav }}$, (scaled by the maneuver load factor $N$, and accounting for both the weight of the wing structure and the lumped masses in Figure 2) and thrust loading, $\boldsymbol{F}_{\text {thrust }}$, from the engine. Aerodynamic forces are written as $q \cdot \boldsymbol{Q} \cdot \boldsymbol{C}_{p}$, where $\boldsymbol{C}_{p}$ is a vector of differential pressure coefficients acting on each panel of the vehicle, $\boldsymbol{Q}$ is an interpolation function derived from FPS, and $q$ is the dynamic pressure. Commanded quasi-steady actuation rotation inputs, $\boldsymbol{\theta}_{i n}$, are applied to the finite element model (specifically as hinge moments applied to each control surface spring) via the matrix $\boldsymbol{Q}_{H M}$.

The second row of Eq. 1 is the aerodynamic analysis, where $\boldsymbol{D}$ is the matrix of aerodynamic influence coefficients (AIC), with a symmetric aerodynamic condition about the centerline of the airplane in Figure 3 . This equation is driven by several terms: downwash due to angle of attack $\left(\boldsymbol{L}_{\alpha} \cdot \alpha\right.$, where $\boldsymbol{L}_{\alpha}$ is a linear operator that converts the scalar angle of attack into a downwash at each panel), elevator deflection, $\boldsymbol{L}_{\delta} \cdot \delta$, built in camber/twist of the wing and tail jig shapes, $\boldsymbol{L}_{j i g}$, and downwash induced by structural wing deformation. This latter term is written as $\boldsymbol{P} \cdot \boldsymbol{x}$, where $\boldsymbol{P}$ is a second interpolation function, also derived from FPS-based methods.

Trim equations are written in the $3^{\text {rd }}$ and $4^{\text {th }}$ rows of Eq. $1: q \cdot \boldsymbol{S}_{L}$ and $q \cdot \boldsymbol{S}_{m}$ convert the differential pressure vector, $\boldsymbol{C}_{p}$, into a total aerodynamic lift and aerodynamic pitching moment (about the aircraft center of gravity). Lift must offset the total weight of the vehicle $(N \cdot W)$, and the net pitching moment must be zero.

The second type of load case considered here is an inertial-only load case, with neither aeroelasticity, trimming mechanisms, nor control surface actuation involved. This is meant to emulate landing loads or taxi bump loads, with wing deformation simply computed by:

$$
\boldsymbol{K} \cdot \boldsymbol{x}=N \cdot \boldsymbol{F}_{\text {grav }}
$$

\section{B. Stresses}

Having solved for the wing deformation in Eqs. 1 and 2, stresses and strains can be computed for each static load case, and a knock-up (safety) factor is applied to each elemental stress value. A range of safety factors are considered for the cases in this paper, up to a value of 1.5. The von Mises failure function is then computed for each finite element. The spar, rib, and skin structures are divided into patches (seen in Figure 1, with a total of 237 patches), and the Kreisselmeier-Steinhauser (KS) function [19] is used to compress all of the elemental failure function values within a given patch into a single metric. If all of the stress values within a patch are within their failure envelope, the KS function for that patch will be less than one.

\section{Panel Buckling}

After the stress analysis, buckling analyses are run for each stiffened panel in the upper and lower surface skins. Following Refs. [20] and [21], each buckling analysis is conducted with a Rayleigh-Ritz method (assumed buckling modes). Both global buckling of a stiffened panel (bordered by ribs and spars) and local buckling between each stiffener is computed, where simply supported boundary conditions are used for both scenarios. The buckling 
equation is given as:

$$
\left(\boldsymbol{K}_{s}+\mu_{n} \cdot \widetilde{\boldsymbol{K}}\right) \cdot \boldsymbol{v}_{n}=\mathbf{0}
$$

where $\boldsymbol{v}_{n}$ is the eigenvector (vector of modal amplitudes) associated with the $n^{\text {th }}$ eigenvalue $\mu_{n}$. The panel stiffness matrix, $\widetilde{\boldsymbol{K}}$, is based upon the stiffness properties defined at the wing level. Smeared stiffness properties are necessarily used for global panel buckling, whereas un-smeared properties are used for local inter-stringer buckling. The panel's geometric stiffness matrix, $\boldsymbol{K}_{s}$, is assembled with element stresses computed from Eqs. 1 or 2.

An eigenvalue, $\mu_{n}$, greater than one indicates that, for the trimmed aeroelastic state, the panel has buckled. Each buckling computation for a given stiffened panel is compressed into a single value using a KreisselmeierSteinhauser function. For a panel with 3 stringers, 4 local buckling computations are performed, along with 1 global buckling computation. Since three modes $(n=3)$ are computed for each eigenproblem, a total of 15 eigenvalues are compressed into a single KS function for this example.

\section{VCCTEF Failure Mechanisms}

Allowable hinge moments for each actuator are prescribed and fixed prior to aeroelastic analysis and optimization. These peak values are estimated from worst-case estimates from Ref. [15], and are also used to estimate the mass of each actuator, as described above. Actual hinge moments incurred during a static maneuver load are computed from the aerodynamic and inertial moments applied to each torsional control surface spring. The ratio of this value to the allowable value must be less than one: each of these hinge moment ratios for a given load case is compressed into a single KS function. Similarly, the peak extensional strain is computed within each elastomeric membrane sheet (connecting spanwise adjacent control surface segments), and the ratio of these strains to some allowable value are also compressed into a KS function, for a given load case.

\section{E. Design Variables and Analytical Sensitivities}

Structural design variables for static aeroelastic optimization include shell thickness, stiffener thickness, and stiffener height for each design patch. In the event that VCCTEF-based maneuver load alleviation is utilized, the vector of actuation inputs, $\boldsymbol{\theta}_{\text {in }}$, are also used as design variables (one set per load case), alongside the structural variables.

Derivatives of the static aeroelastic response (stress, buckling, hinge moment, and elastomer strain aggregation parameters for each load case) with respect to all design variables are computed with the adjoint method. This method is advantageous as the number of constraints is consistently less than the number of design variables, for a given load case [22].

\section{Static Aeroelastic Optimization}

The optimal distribution of patch-based skin thickness, stiffener thickness, and stiffener height variables (using the rib, spar, and skin design patches in Figure 1), as well as the quasi-steady actuation-input design variables $\left(\boldsymbol{\theta}_{\text {in }}\right)$, are found that minimize the wing mass (based on the volume of the finite element model) subject to a variety of constraints:

$$
\begin{aligned}
& \underset{\boldsymbol{q}}{\min } \text { mass } \\
& \text { s.t. } \begin{cases}\mathbf{0} \leq \boldsymbol{q} \leq \mathbf{1} & \\
K S_{\sigma, i} \leq 1 & i=1, \ldots,\left(N_{\sigma} \cdot N_{L}\right) \\
K S_{\mu, i} \leq 1 & i=1, \ldots,\left(N_{\mu} \cdot N_{L}\right) \\
K S_{H M, i} \leq 1 & i=1, \ldots, N_{L} \\
K S_{e, i} \leq 1 & i=1, \ldots, N_{L} \\
K S_{t / t_{s}} \leq 1 & \\
K S_{A R} \leq 1 & \end{cases}
\end{aligned}
$$

where $\boldsymbol{q}$ are the design variables that have been appropriately normalized between 0 and 1 . Dimensionally, shell thicknesses are allowed to range between $3 \mathrm{~mm}$ and $30 \mathrm{~mm}$, stiffener thicknesses between $2.5 \mathrm{~mm}$ and $150 \mathrm{~mm}$, and stiffener heights between $30 \mathrm{~mm}$ and $150 \mathrm{~mm}$ (except for vertical stiffeners on the ribs and spars, whose lower height bound is set to $75 \mathrm{~mm}$ : this higher value helps prevent local vibration modes in these areas). 
Input design variables for control surface rotation are bounded between $\pm 7^{\circ}$. If, for a given span-station, all three VCCTEF segments are set to $7^{\circ}$, the rotation of the downstream segment relative to the wing will be $21^{\circ}$. It is further noted that $\boldsymbol{\theta}_{\text {in }}$ are commanded inputs, which will deviate from the actual control surface rotations, depending on the relative magnitudes of the aerodynamic/inertial resistive hinge moments and the torsional spring constant.

In Eq. $4, N_{L}$ is the number of static load cases, $K S_{\sigma}$ are the stress aggregation parameters ( $N_{\sigma}$ per load case), $K S_{\mu}$ are the buckling aggregation parameters $\left(N_{\mu}\right.$ per load case), $K S_{H M}$ are the hinge moment aggregation parameters ( 1 per load case), and $K S_{e}$ are the elastomer strain aggregation parameters ( 1 per load case). It is desired that, for a given design patch, the shell thickness not be greater than the stiffener thickness by $2.5 \mathrm{~mm}$. These constraints are normalized to 1 for each design patch, and then all aggregated into a single metric, $K S_{t / t_{S}}$. Similarly, the aspect ratio of each stiffener should not be greater than 15 , which is compressed into the single constraint, $K S_{A R}$.

Five static load cases are considered here, summarized in Table 1. Load cases 1 and 3 are closed-loop, in the sense that the VCCTEF system is used for maneuver load alleviation. The knock-up factor (or safety factor) is set to the commonly-used value of 1.5 for these cases. Load cases 2 and 4 are open-loop, as VCCTEF is not used here. These cases will be rarely encountered in flight (only if the VCCTEF system is not active), and so a lower knock-up factor may potentially be used, at the expense of greater overall risk. Load cases 1-4 are computed with the static aeroelastic Eq. 1; case 5 is inertial-only, and computed with Eq. 2.

Table 1. Summary of static aeroelastic load cases.

\begin{tabular}{|c|c|c|c|c|c|}
\hline $\begin{array}{c}\text { Load } \\
\text { Case }\end{array}$ & $\begin{array}{c}\text { Load } \\
\text { Factor }\end{array}$ & Mach & Altitude & $\begin{array}{c}\text { Safety } \\
\text { Factor }\end{array}$ & $\begin{array}{c}\text { VCCTEF } \\
\text { Status }\end{array}$ \\
\hline 1 & 2.5 & 0.85 & $10,000 \mathrm{ft}$ & 1.5 & closed-loop \\
\hline 2 & 2.5 & 0.85 & $10,000 \mathrm{ft}$ & various & open-loop \\
\hline 3 & -1 & 0.6 & $0 \mathrm{ft}$ & 1.5 & closed-loop \\
\hline 4 & -1 & 0.6 & $0 \mathrm{ft}$ & various & open-loop \\
\hline 5 & 3 & - & - & 1.5 & open-loop \\
\hline
\end{tabular}

For a single wing, all load cases are run with full fuel in the wing $(45,000 \mathrm{~kg})$, a 7,400 kg engine, $300 \mathrm{~kg}$ of lumped mass along the leading-edge, and a 2,500 kg VCCTEF system (mass of all actuators and control surface segments). Non-modeled mass (fuselage, payload, etc.) for the half-vehicle is fixed at $80,000 \mathrm{~kg}$. Typical singlewing structural mass values (the objective function in Eq. 4) range from 10,000-15,000 kg, so the TOGW for the entire vehicle is roughly $300,000 \mathrm{~kg}$. This value compares well to publically-available data for transports of similar size to the uCRM [23].

Each set of structural design variables (shell thickness, stiffener thickness, and stiffener height) is passed through a linearly-decaying cone-shape filter [24] in order to prevent the difference in stiffness between adjacent patches from being too large. The same spatial filter is utilized for the input actuation variables, to prevent large discrepancies between the rotation of adjacent control surface segments: the elastomer membrane strain constraint, $K S_{e}$, will also help prevent this. A linear matrix relationship can be built between the un-filtered design variables (which are directly created by the optimizer), and the filtered design variables (which are used to construct the aeroelastic model). All gradient-based optimization problems are solved with the Globally-Convergent Method of Moving Asymptotes tool (GCMMA) [25].

First, the knock-up (safety) factor of the open-loop cases 2 and 4 is set to one. This stipulates that these openloop cases, if encountered (i.e., if the VCCTEF system is inactive), will not experience structural failure, but no safety factor is accounted for either. The closed-loop safety factor is set (in all cases in this paper) to 1.5: this is a much harder set of structural constraints for the optimizer to satisfy. On the other hand, the closed-loop load cases can take advantage of the maneuver load alleviation afforded by the optimized VCCTEF system.

The optimal result is shown in Figure 4, in terms of the shell thickness, smeared stiffener thickness, and smeared stiffener height of each design patch. The optimizer places the majority of the material in the upper and lower surface skins, with stiffness tapered towards the lower bound at the wing tip, due to the drop in bending moment. Rib design variables are all pushed to the lower bound, as are many of the spar variables, with the exception of the thick trailing-edge root spar. Crushing loads are not included here, which may drive the optimizer to strengthen the rib members. The mass (objective function) of this structure in $9,138 \mathrm{~kg}$, which is based on the volume of the finite element model (including smeared stiffeners), and does not include the lumped mass or control surfaces seen in Figure 2. 


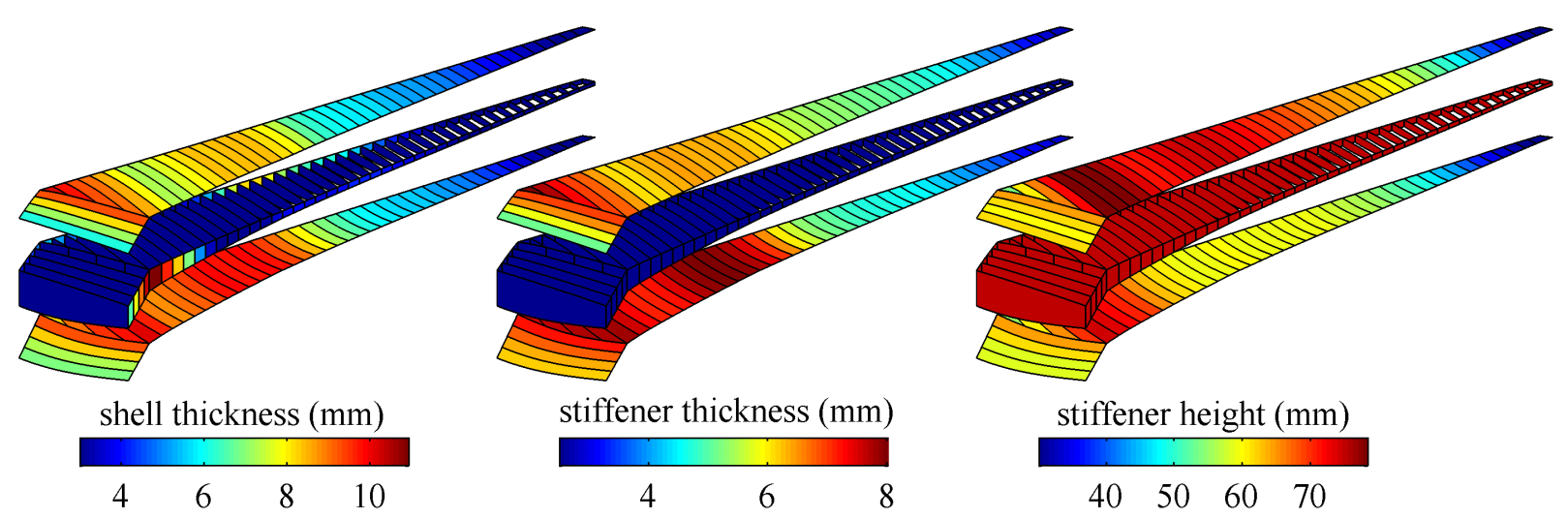

Figure 4. Optimal structural design variables for the static aeroelastic optimization problem.

Wing deflections and element-level von Mises failure indices are shown for this structure in Figure 5, during a 2.5-g pull-up maneuver, with and without VCCTEF-based maneuver load alleviation (cases 1 and 2 from Table 1). $\mathrm{KS}$ buckling parameters along the upper surface wing skin are shown for these same cases in Figure 6 . It is noted that the stress data in Figure 5 is not explicitly provided to the optimizer, but is first compressed into $K S_{\sigma}$ functions for each segment (along with stresses in the stiffeners, which are not shown). The buckling data in Figure 6 are values of $K S_{\mu}$, and therefore are provided to the optimizer in the form shown. As discussed above, each buckling failure index $\left(K S_{\mu}\right)$ is an aggregation of each global and local (inter-stringer) eigenvalue for a given stiffened panel. For both stress data and buckling data, a value greater than one would be an infeasible aeroelastic constraint, and is not seen in either plot. Similar data exists for the remaining three load cases in Table 1, but is not shown here.

As would be expected, wing deflections in Figure 5 are larger without maneuver load alleviation (open-loop), and high stresses are spread throughout a larger portion of the wing skins. For both cases in the figure, however, peak stress concentration occurs at the side-of-body connection along the trailing-edge. The contoured values in Figure 5 are stress indices with the knock-up factor included. As such, the actual peak von Mises stresses for the closed-loop case are yield stress $\left(\sigma_{Y}\right)$ divided by a factor of 1.5: for the open-loop case, peak stresses are nearly equal to $\sigma_{Y}$. The same correction scheme is applied to the buckling factors of Figure 6 , which are seen to be active for many panels throughout the wing from root to tip, and therefore an important design driver. Panel buckling is inactive for the lower surface skin panels during this $2.5 \mathrm{~g}$ maneuver, (these panels are in tension), but becomes important during the $-1 \mathrm{~g}$ push-over maneuver and the $3 \mathrm{~g}$ inertial maneuver, neither of which is shown.

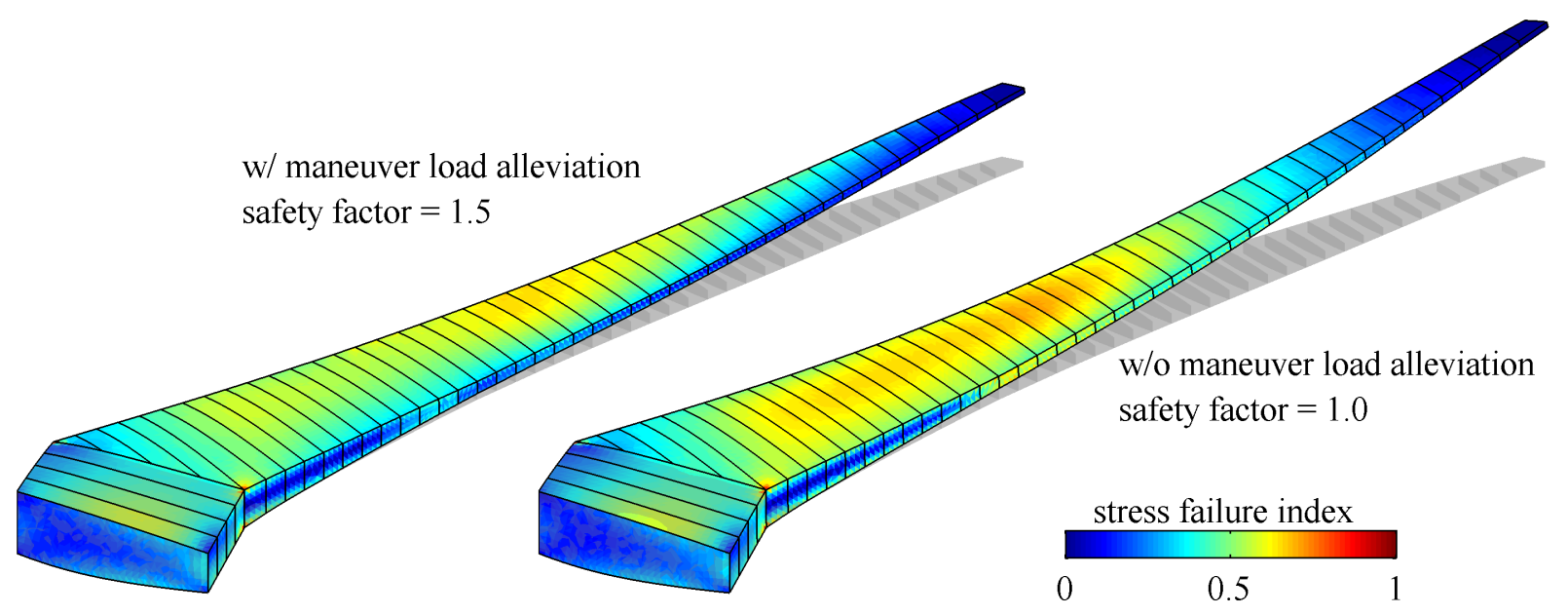

Figure 5. Wing deflection and stress-based failure indices during a $2.5 \mathrm{~g}$ pull-up: with (left) and without (right) VCCTEF maneuver load alleviation.

The quasi-steady actuation-input design variables, $\boldsymbol{\theta}_{\text {in }}$, corresponding to this optimal structure can be seen in Figure 7. VCCTEF actuation is more pronounced for the $2.5 \mathrm{~g}$ maneuver than the $-1 \mathrm{~g}$ maneuver, as the stresses for 
this case are strong design drivers (buckling constraints are active for both cases, however). The expected behavior during the pull-up maneuver is for the optimizer to use negative control surface rotations outboard. This would reduce the maneuver bending moment on the wing, and allow the optimizer to remove material near the root without violating stress or buckling constraints. This outboard wash-out would be counteracted by inboard wash-in (positive rotations) to maintain trim, which can be done without increasing structural loads owing to the short moment arm. This overall optimal behavior is noted for segment 1 (upstream, closest to the wing), and to a lesser degree for segment 2, but the downstream segment 3 follows an opposite spatial pattern.

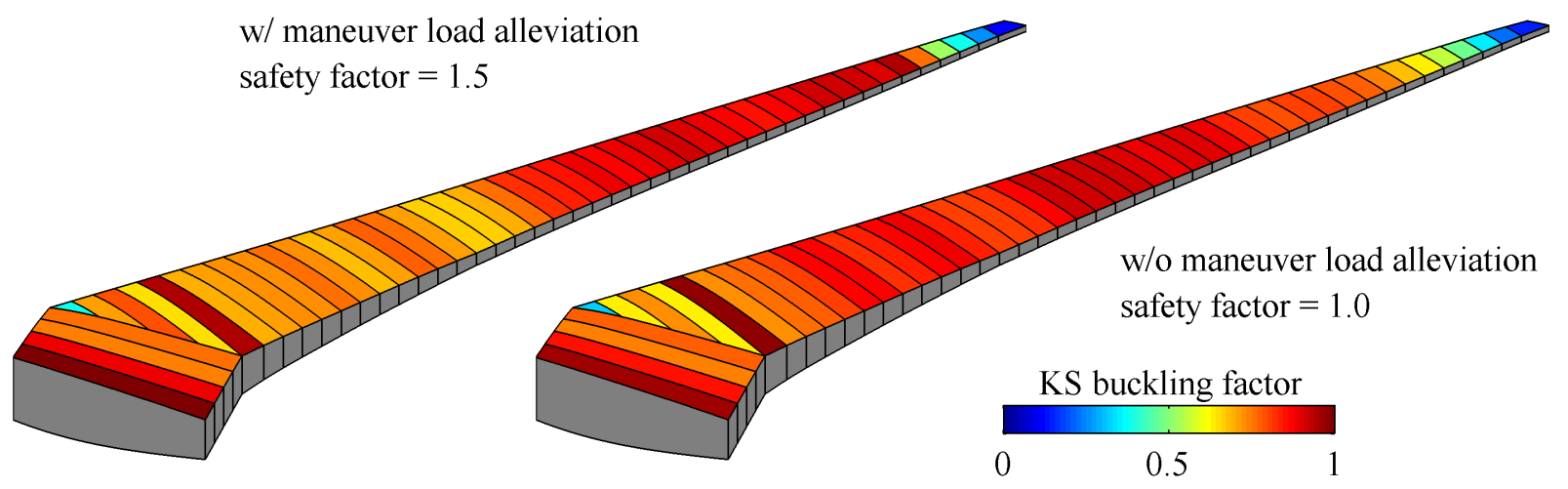

Figure 6. Buckling failure indices (KS functions) for each stiffened panel during a 2.5g pull-up: with (left) and without (right) VCCTEF maneuver load alleviation.

The contrarian behavior of segment 3 along the trailing-edge of the wing, for the $2.5 \mathrm{~g}$ maneuver in Figure 7 , is likely driven by the need to satisfy hinge moment constraints $\left(K S_{H M}\right.$ in Eq. 4) for the 2 upstream segments, particularly segment 1 . The optimizer is using segment 3 in much the same way as a trim or balance tab. Hinge moments are plotted in Figure 8, where the dashed lines indicate the allowable values. As noted above, these peak allowable hinge moments are prescribed and fixed prior to optimization. At the root, $\pm 25,000 \mathrm{~N} \cdot \mathrm{m}, \pm 12,500 \mathrm{~N} \cdot \mathrm{m}$, and $\pm 2,500 \mathrm{~N} \cdot \mathrm{m}$ are allowed for segments 1,2 , and 3, respectively. A linear variation is utilized down to the tip, where values of $\pm 5,000 \mathrm{~N} \cdot \mathrm{m}, \pm 2,500 \mathrm{~N} \cdot \mathrm{m}$, and $\pm 750 \mathrm{~N} \cdot \mathrm{m}$ are allowed.
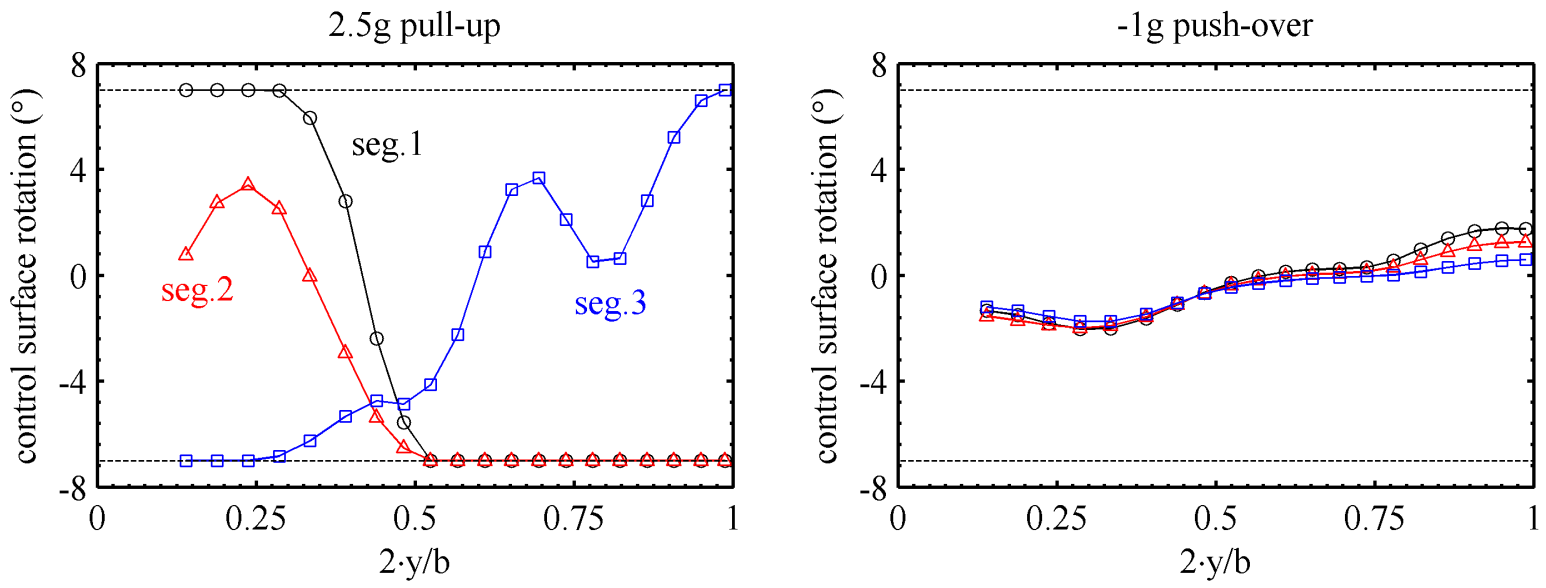

Figure 7. Commanded VCCTEF actuation for the two aeroelastic load cases.

Due to the conservative nature of the aggregation constraint, an active $K S_{H M}$ (i.e., equal to one) will prevent the hinge moment values from being exactly equal to the allowable values in Figure 8 at a given span station. Segments for which the hinge moment is close to the allowable value, however (such as for segment 1 at the wing root, or segment 3 at the wing tip), would indicate the need for a bigger actuator. Conversely, the overall vehicle weight would benefit from smaller actuators for the multiple VCCTEF segments for which these hinge moment constraints are inactive. An interesting extension of this work may be to include VCCTEF actuator sizing variables in the overall design variable list of Eq. 4, to minimize a weighted sum of structural and actuator weight. 


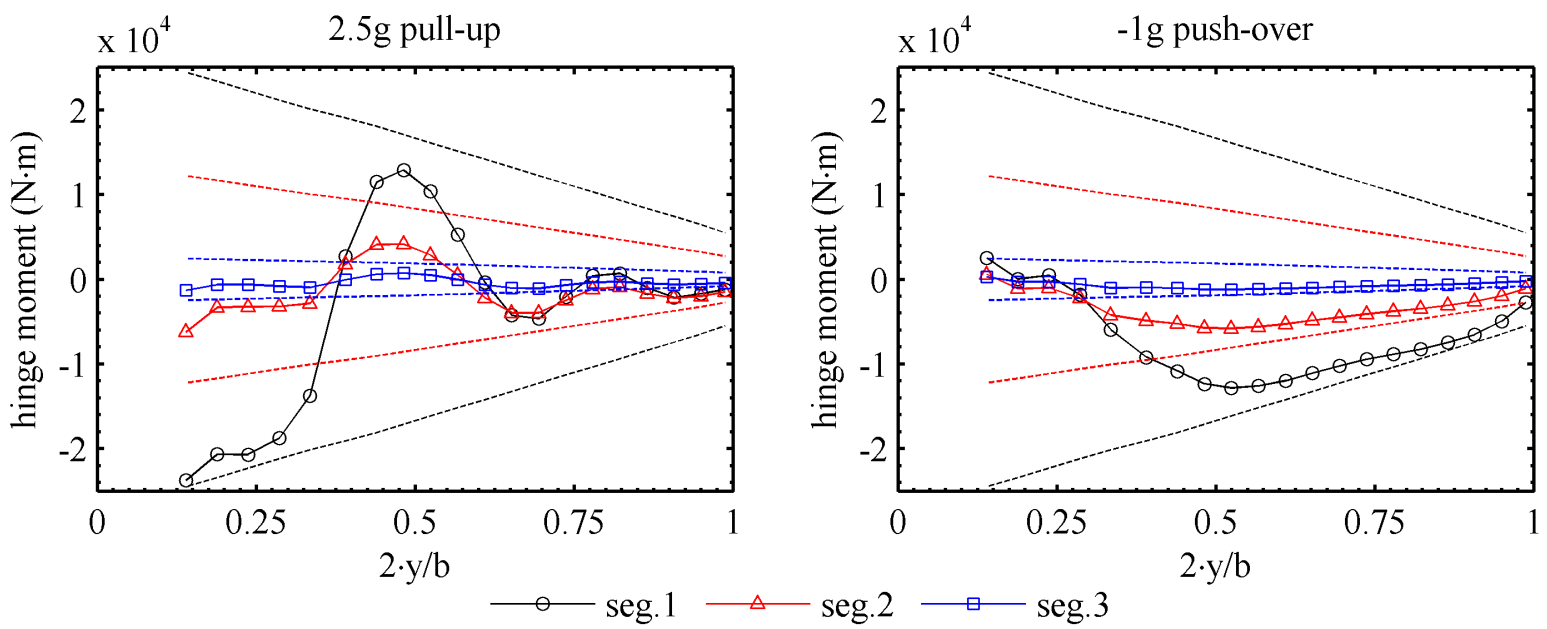

Figure 8. Computed hinge moments (symbols) and allowable hinge moments (dashed lines) for the two aeroelastic load cases.

Results to this point have utilized an open-loop safety factor (for load cases 2 and 4 in Table 1) of 1, and a closed-loop safety factor (cases 1 and 3 ) of 1.5. It is of interest to understand how the optimizer reacts to changes in the open-loop factor, in terms of the minimum available structural mass. These results are shown in Figure 9: higher open-loop factors result in a substantial increase in wing mass, as the constraint becomes harder to satisfy, but also result in an implicitly lower level of risk should the VCCTEF system be inactive during flight.

One extreme of the plot is where the open-loop safety factor is set to the same 1.5 value as the closed-loop cases. Here, the VCCTEF system becomes meaningless, as both open- and closed-loop load cases are of equal importance to the optimizer, but the former will always be critical, as it does not benefit from VCCTEF. The same optimal mass would be obtained if only open-loop load cases were considered, and $\boldsymbol{\theta}_{\text {in }}$ removed from the list of design variables. The other extreme of Figure 9 is when the open-loop safety factor is set to values below one. In this case, the stress and buckling constraints associated with the open-loop cases become inactive, and the optimal mass is no longer a function of the open-loop safety factor.

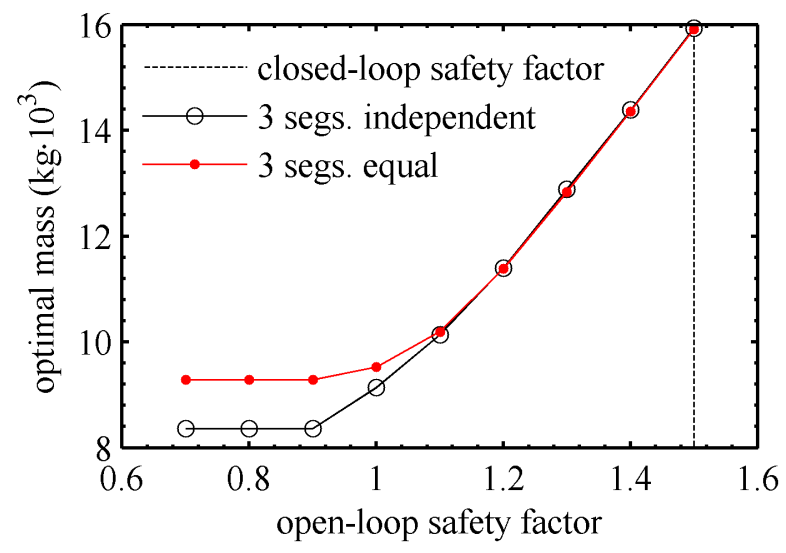

Figure 9. Optimal wing mass as a function of the open-loop knock-up factor.

A common approximation in VCCTEF studies [11] is to limit the input rotation of the 3 control surface segments at a given span station to be the same value (such that the 3 segments form a circular arc), so as to limit the number of design variables/parameters. This work utilizes analytical adjoint derivatives, and so there is no need to decrease the design variable set, but it is of interest to understand the aeroelastic performance lost with this approximation. It can be seen in Figure 9 that, above an open-loop safety factor of 1.1, either parameterization (if the 3 segments are equal, or are independently articulated) will give the same optimal wing mass. This is because the open-loop load cases (which are unaffected by the choice of VCCTEF articulation) are strong overall design 
drivers, and the $\boldsymbol{\theta}_{\text {in }}$ design variables are not pushed to the side limits of $\pm 7^{\circ}$.

Below an open-loop factor of 1.1, however, differences between the two parameterizations are seen in Figure 9, as the closed-loop constraints become more important and actuation design variables reside at the side limits (seen in Figure 7, for example). In the extreme case, wing structures optimized with a reduced VCCTEF parameterization are $11 \%$ heavier than those that optimize each segment independently. Optimal actuation for both cases is shown in Figure 10 for the $2.5 \mathrm{~g}$ pull-up maneuver, where the deflections on the left correspond to those in Figure 7. The reduced parameterization obviously precludes the optimizer from using the third segment as a balance tab, and so inboard actuation cannot be utilized without violating the hinge moment constraints. It is expected that if the hinge moment constraints were made easier to satisfy, the differences between the two parameterizations in Figure 9 would decrease.

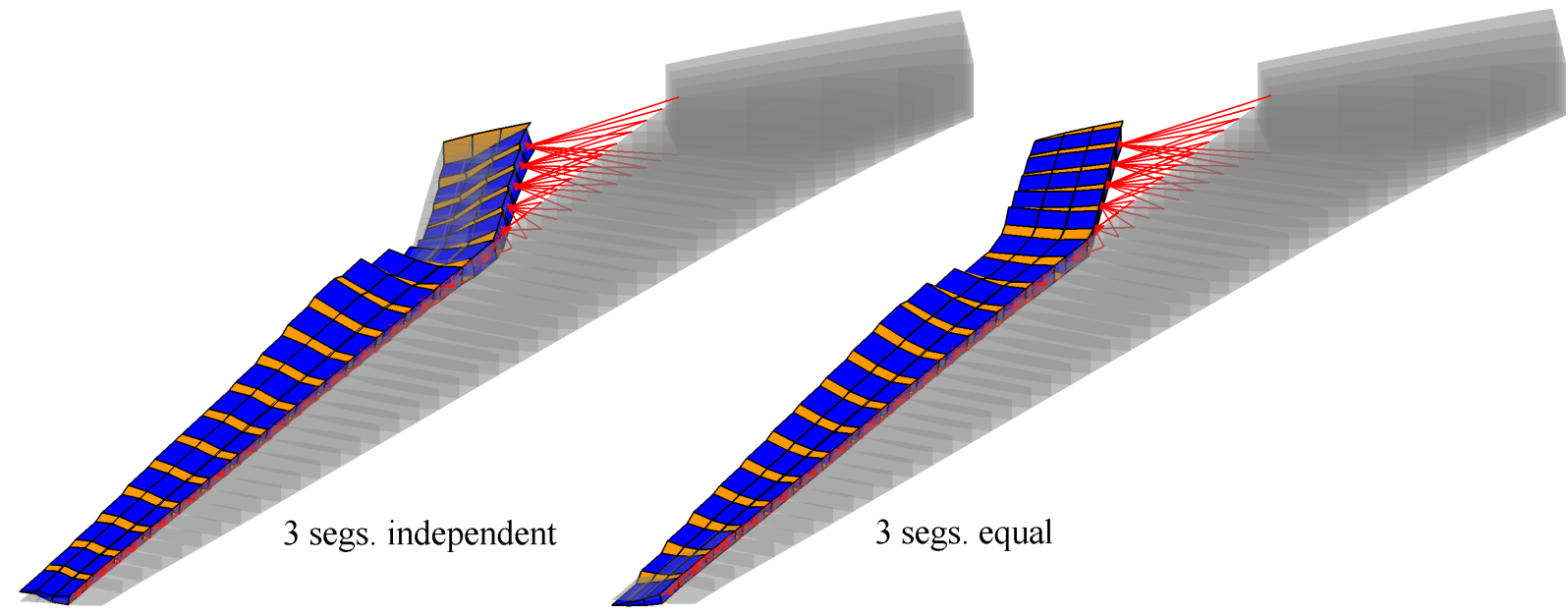

Figure 10. Optimal VCCTEF actuation (scaled by a factor of 3) during the $2.5 \mathrm{~g}$ maneuver.

\section{Dynamic Aeroelastic Optimization}

The exercises to this point have only included static aeroelastic constraints computed across a variety of trimmed maneuvers: stresses and buckling within the wingbox, hinge moments and elastomer strains within the VCCTEF actuation system. This optimization problem (Eq. 4) will now be augmented with flutter constraints. Flutter margins of $15 \%$ (beyond the flight envelope) as measured by equivalent air speed are typically required. Open-loop flutter constraints (which do not utilize VCCTEF actuation) may be relaxed below this value, but closedloop constraints (which do) are maintained at $15 \%$, subject to a control cost constraint.

As noted above, only the third row of VCCTEF segments (electric drive motors) may be dynamically actuated for flutter suppression. Any oscillatory actuation obtained here may, in theory, be superimposed upon the quasisteady VCCTEF deflections found in the previous section. Flutter mechanisms are sought over a range of flight conditions: however, those conditions will not, in general, match the conditions (altitude) used for the static maneuver loads in Table 1.

\section{A. Open-Loop Flutter}

Aerodynamic analyses are computed using the doublet lattice method in the frequency domain. Open-loop flutter constraints could also be computed in the frequency domain (using the $\mathrm{p}-\mathrm{k}$ method), but are instead computed in the time domain (using the p-method). This is to provide a direct comparison with the results of the next section, where closed-loop control requires state-space modeling. The development of a state-space aeroelastic flutter model is done with well-known methods [1], and will only be briefly described here.

First, mode shapes (eigenvectors) of the flexible wing with rigidly-attached VCCTEF control surfaces are computed. Next, twenty control modes (one for each dynamically-actuated control surface) are computed as Ritz vectors, where each vector is zero except for a unit rotation of the corresponding control surface torsional-hinge spring. The flexible mode shapes and the Ritz vectors are combined into a single modal matrix, and properly orthogonalized [26]. These modes are used to compute reduced mass, stiffness, and damping matrices, as well as generalized aerodynamic forces at tabulated reduced frequencies and Mach numbers. Frequency domain aerodynamics are converted to the time domain using the Roger approximation [27].

The final, linear time-invariant system is written as: 


$$
\left\{\begin{array}{c}
\dot{\boldsymbol{x}}_{m} \\
\ddot{\boldsymbol{x}}_{m} \\
\dot{\boldsymbol{x}}_{A}
\end{array}\right\}=\left[\begin{array}{ccc}
\boldsymbol{I} & \mathbf{0} & \mathbf{0} \\
\mathbf{0} & \overline{\boldsymbol{M}}-\frac{q \cdot b^{2}}{U^{2}} \cdot \boldsymbol{A}_{2} & \mathbf{0} \\
\mathbf{0} & \mathbf{0} & \frac{b}{U} \cdot \boldsymbol{I}
\end{array}\right]^{-1} \cdot\left[\begin{array}{ccccc}
\mathbf{0} & \boldsymbol{I} & \mathbf{0} & \cdots & \mathbf{0} \\
-\overline{\boldsymbol{K}}+q \cdot \boldsymbol{A}_{0} & -\overline{\boldsymbol{C}}+q \cdot \boldsymbol{A}_{1} & q \cdot \boldsymbol{A}_{2} & \cdots & q \cdot \boldsymbol{A}_{n+2} \\
\mathbf{0} & \frac{b}{U} \cdot \boldsymbol{I} & -\gamma_{1} \cdot \boldsymbol{I} & \cdots & \mathbf{0} \\
\vdots & \vdots & \vdots & \ddots & \vdots \\
\mathbf{0} & \frac{b}{U} \cdot \boldsymbol{I} & \mathbf{0} & \cdots & -\gamma_{n} \cdot \boldsymbol{I}
\end{array}\right] \cdot\left\{\begin{array}{c}
\boldsymbol{x}_{m} \\
\dot{\boldsymbol{x}}_{m} \\
\boldsymbol{x}_{A}
\end{array}\right\}
$$

where $b$ is half the mean aerodynamic chord, and $\overline{\boldsymbol{M}}, \overline{\boldsymbol{K}}$, and $\overline{\boldsymbol{C}}$ are the reduced mass, stiffness, and damping matrices. $\boldsymbol{A}_{i}$ are the generalized aerodynamic forces resulting from the rational function approximation, and $\gamma_{i}$ are the corresponding lag roots $\left(n\right.$ total). $\boldsymbol{x}_{m}$ is the vector of modal amplitudes (wing deformation and control surface rotation), and $\boldsymbol{x}_{A}$ is the vector of aerodynamic states. No rigid body modes are considered here: the wing is fullyclamped at its centerline and pinned at its root, as above.

Eigenvalues of Eq. 5 are given by $s=g+i \cdot \omega$. For a given Mach number, these eigenvalues may be tracked across a range of matched point equivalent air speeds. Speeds at which a given eigenvalue crosses into the right-half plane $(g=0)$ are flutter points. A flutter constraint is formulated by imposing upper limits on the damping $(g)$ of each mode, at all equivalent air speeds $\left(U_{E A S}\right)$ of interest [28][29]. Specifically, each damping value is constrained to lie beneath the piecewise polynomial:

$$
g \leq \begin{cases}g^{*} \cdot\left(3 \cdot U^{*} \cdot U^{2}-2 \cdot U^{3}\right) /\left(U^{*}\right)^{3} & 0 \leq U_{E A S}<U^{*} \\ s \cdot\left(U_{E A S}-U^{*}\right)^{2}-g^{*} & U_{E A S} \geq U^{*}\end{cases}
$$

The minimum allowable flutter equivalent air speed is $U^{*}$, computed from the desired flutter margin and the flight envelope (using a $185 \mathrm{~m} / \mathrm{s}$ dive speed). The quadratic scaling parameter $s$ is set to 500 , and the required damping at the flutter speed, $g^{*}$, is set to 0.2 . Using a non-zero $g^{*}$ prevents an optimal structure from having a hump mode with a peak damping of nearly 0 : a theoretically stable situation, but highly non-robust [30].

Critical $U_{E A S}$ points (local minima) of the inequality in Eq. 6 are computed and lumped together into a single Kreisselmeier-Steinhauser constraint. As above, if this constraint is greater than one, then Eq. 6 is not satisfied, and the structure does not meet the required flutter margin. A separate KS constraint is utilized for each considered Mach number. Derivatives of the flutter constraint are computed with eigenvalue gradient techniques. A fixedmode dynamic derivative approach is utilized here, where the derivative of the mode shapes with respect to design variables is neglected for the purposes of gradient computations. Mode shapes are actually updated during every outer iteration of GCMMA, when design gradients are required by the tool as well. During the line-search inner iterations, when gradients are not required, the modes are held fixed. As such, the fixed-mode approximation is exact during the inner iteration's search, but not during the outer iteration.

The optimization problem of the previous section is repeated here, adding the flutter constraint (at $0.85 \mathrm{Mach}$ ) to the static aeroelastic constraints. Both structural design variables (shell thickness, stiffener thickness, stiffener height) and quasi-steady VCCTEF input actuation, $\boldsymbol{\theta}_{i n}$, are again utilized as before. The open-loop safety factor for static stresses and buckling (load cases 2 and 4 in Table 1) is fixed at 1.3 for the remainder of this paper: referencing Figure 9, the optimal mass for this case is $12,885 \mathrm{~kg}$. The mass penalty associated with adding an open-loop flutter constraint to this design is shown in Figure 11. Imposing a 15\% flutter margin requires the optimizer to increase the mass by $24.7 \%$. Margins below zero are unstable within the flight envelope (as no closed-loop mechanism is utilized in this example): an obviously unacceptable result shown only to provide context for the trade-off between structural mass and flutter.

Eigenvalue migration plots (real part) are shown in Figure 12, before and after the 15\% flutter margin constraint is implemented. The static aeroelastic optimum without the flutter constraint (left side of Figure 12) has several fluttering modes, but the critical two are a hump mode that flutters at $149.6 \mathrm{~m} / \mathrm{s}\left(U_{E A S}\right)$ and a more severe instability that flutters at $159.8 \mathrm{~m} / \mathrm{s}$. This is commonly seen for transport aircraft [31], where the primary hump mode is a torsion mode dominated by engine pitch, and the "hard" flutter point is a bend-torsion coupling. The dashed line in these figures is the constraint boundary of Eq. 6. This constraint is satisfied on the right plot of Figure 12, and strongly active, as two modes brush up against the boundary in the vicinity of $U^{*}$ and $g^{*}$.

Optimal structural variables for the case with the 15\% flutter margin are shown in Figure 13, and directly comparable to the structural variables in Figure 4, where no flutter constraint is imposed. Several key topological differences are noted: most predominantly, material is added to the wing tip, a clear attempt to alter the inertial loads via separation of coalescing modal frequencies. This occurs despite the $3 \mathrm{~g}$ inertial load case included in Table 1 , whose severity would presumably increase with added mass at the tip. The buckling and stress values for this load 
case are strongly active, but not violated. Secondly, the thickness of the spars and the height of the stiffeners are increased in the vicinity of the engine attachment points, which is presumably driven by the critical engine-pitch fluttering hump mode.

The optimal quasi-steady VCCTEF actuation, $\boldsymbol{\theta}_{i n}$, for this case is not appreciably different from the cases considered in the previous section, and is not shown here.

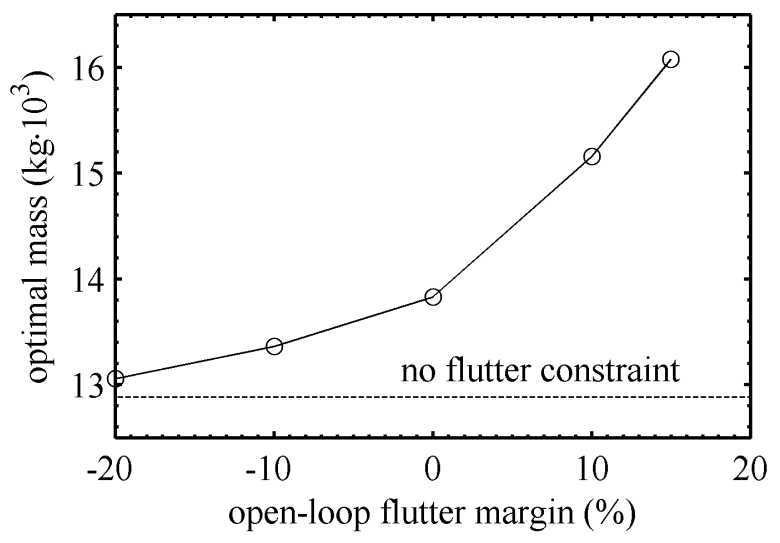

Figure 11. Trade-off curve between the open-loop flutter constraint and the minimum structural mass.
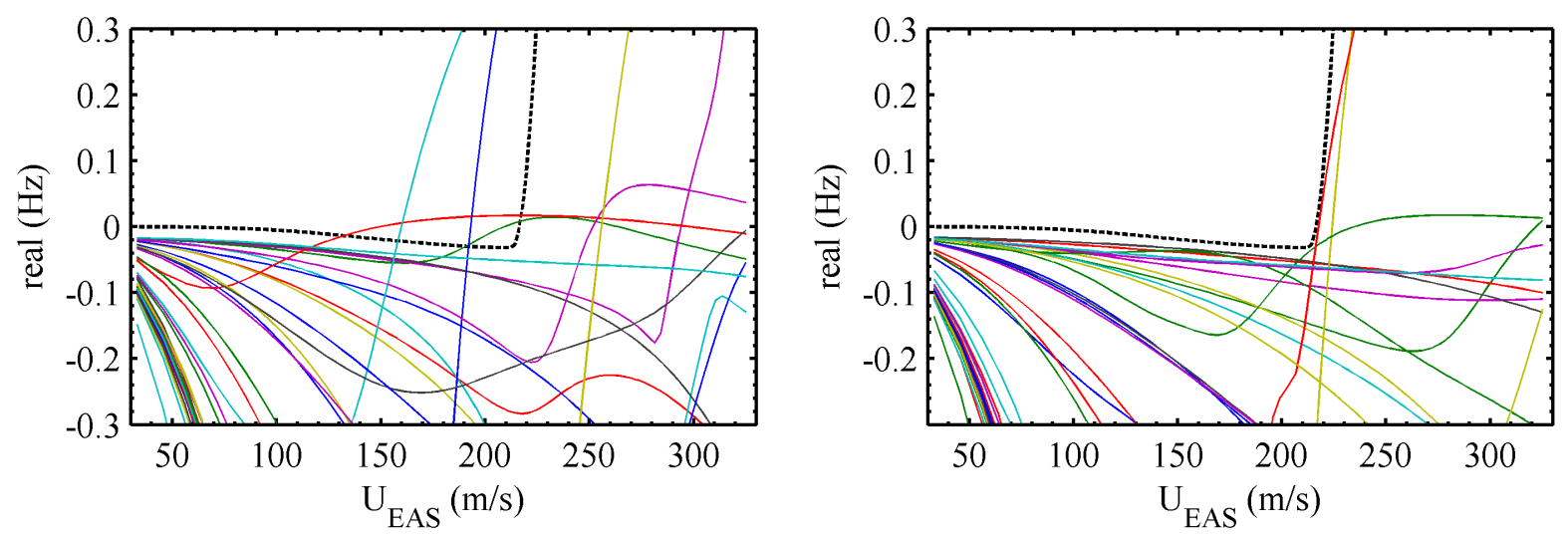

Figure 12. Real-part eigenvalue migration without (left) and with (right) a $15 \%$ flutter margin constraint enforced.

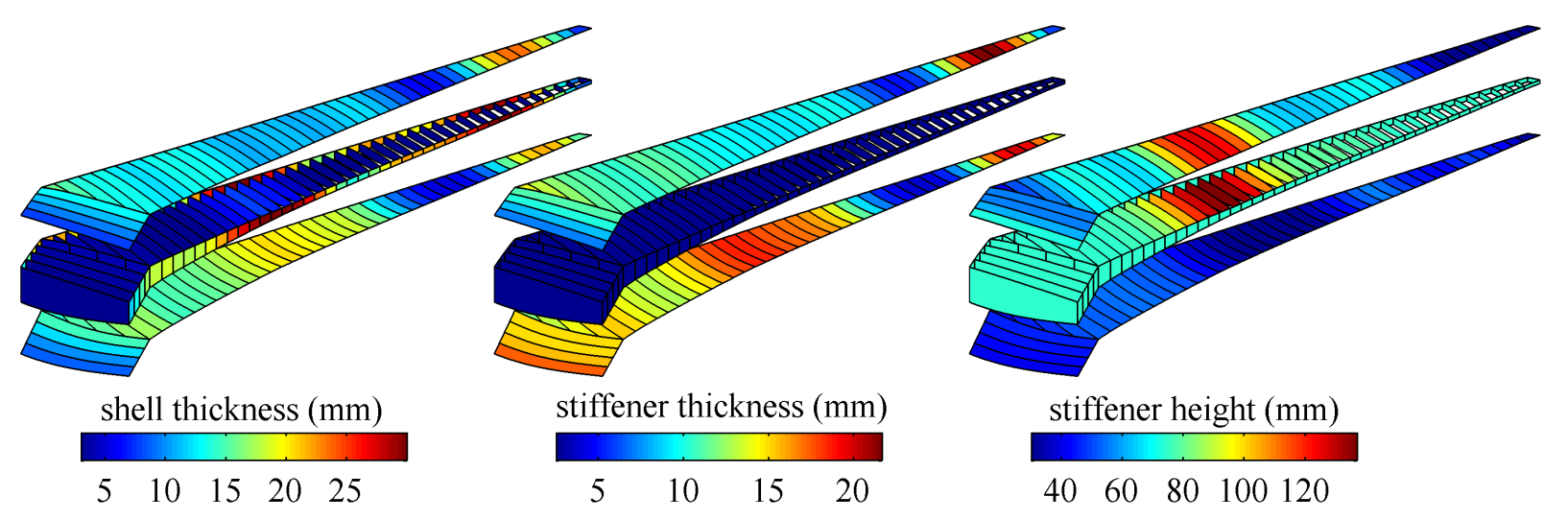

Figure 13. Optimal structural design variables for the optimization problem with static aeroelastic constraints and open-loop flutter constraints. 


\section{B. Closed-Loop Flutter}

The previous section has demonstrated a substantial weight penalty with the addition of an open-loop flutter constraint to a design optimized under static aeroelastic constraints. This section explores the idea of relying on closed-loop control to meet the $15 \%$ margin, and assessing the weight savings from a relaxed open-loop flutter constraint via aeroservoelastic optimization. Ideally, the controller may be able to meet this flutter margin without needing to add extra stiffness (mass) to the wing, though the control cost (system power) must be accounted for [1].

The aeroservoelastic linear time-invariant system is given as:

$$
\dot{\boldsymbol{x}}_{A S E}=\boldsymbol{A} \cdot \boldsymbol{x}_{A S E}+\boldsymbol{B} \cdot \boldsymbol{u}_{A S E}
$$

where $\boldsymbol{x}_{A S E}$ is the state vector from Eq. 5 and $\boldsymbol{A}$ is the matrix from Eq. 5. $\boldsymbol{u}_{A S E}$ is a vector of input control surface rotations (one per electric drive motor, the third row of VCCTEF control surface segments), analogous to the quasisteady $\boldsymbol{\theta}_{\text {in }}$ variables. $\boldsymbol{B}$ is a matrix which converts those commanded rotations into generalized modal forces. The controller used here is a linear quadratic regulator (LQR), where feedback is assumed of the form:

$$
\boldsymbol{u}_{A S E}=-\boldsymbol{K}_{L Q R} \cdot \boldsymbol{x}_{A S E}
$$

The feedback matrix is found which minimizes the performance index [32]:

$$
J=\frac{1}{2} \cdot \int_{0}^{\infty}\left(\boldsymbol{x}_{A S E}^{T} \cdot \boldsymbol{Q} \cdot \boldsymbol{x}_{A S E}+\boldsymbol{u}_{A S E}^{T} \cdot \boldsymbol{R} \cdot \boldsymbol{u}_{A S E}\right) \cdot d t
$$

where $\boldsymbol{Q}$ is the state weighting matrix, and $\boldsymbol{R}$ is the control weighting matrix.

Two types of controller-centric design variables are considered. The first are the members of the diagonal control weighting matrix, $\boldsymbol{R}$, (one per electric drive motor), which are passed through a spatial filter in order to maintain a smooth shape along the trailing-edge, much like the quasi-steady $\boldsymbol{\theta}_{\text {in }}$ design variables. Upper and lower bounds are necessarily placed on the $\boldsymbol{R}$ design variables. Very large values (relative to $\boldsymbol{Q}$ ) penalize the control action, and so instabilities are arrested slowly. Small values of $\boldsymbol{R}$ are indicative of inexpensive control, where instabilities can be damped out quickly. The matrix $\boldsymbol{Q}$, a fixed quantity for all cases, is computed from a unitweighting of the displacement of eight finite element nodes uniformly distributed throughout the wing: two chordwise and four spanwise.

The second type of controller design variable is the flight condition (namely, the equivalent air speed $U_{L Q R}$ ) at which the feedback matrix, $\boldsymbol{K}_{L Q R}$, is computed. Gain scheduling is the preferred long-term solution for active flutter control of transport wings [33], but for the conceptual studies provided here, a single control law is used for the entire flight envelope. However, the optimizer is able to choose the flight condition at which the controller is designed. A similar type of design variable is utilized in Refs. [4] and [5]. Large values of the $U_{L Q R}$ control design point (relative to the open-loop flutter point) will stabilize the system at $U_{L Q R}$, as guaranteed by the LQR methodology [32], but the control cost, $J$, will be high. Small values of $U_{L Q R}$ may not provide a closed-loop flutter point measurably better than the open-loop point, but the control cost will be low.

The aeroservoelastic optimization problem solved in this section is to minimize mass, subject to the static aeroelastic stress $\left(K S_{\sigma}\right)$ and buckling $\left(K S_{\mu}\right)$ constraints, VCCTEF hinge moment $\left(K S_{H M}\right)$ and elastomer strain $\left(K S_{e}\right)$ constraints, stiffener geometry $\left(K S_{t / t_{s}}\right.$ and $\left.K S_{A R}\right)$ constraints, an open-loop flutter constraint, a closed-loop flutter constraint, and a limit on the maximum allowable control cost $(J)$. The closed-loop constraint is always set to a margin of $15 \%$, but lower open-loop margins are considered, as discussed above. Both margins are evaluated at a single Mach number of 0.85 .

Design variables include structural variables (shell thickness, stiffener thickness, stiffener height), quasi-steady VCCTEF input rotation variables $\left(\boldsymbol{\theta}_{i n}\right)$, the control weighting terms $(\boldsymbol{R})$, and the controller design point $\left(U_{L Q R}\right)$. Gradients of the feedback matrix, $\boldsymbol{K}_{L Q R}$, and the performance index, $J$, are computed using methods in Ref. [34]. Once the $\boldsymbol{K}_{L Q R}$ derivatives are found, derivatives of the closed-loop flutter constraint are computed with the same eigenvalue sensitivity methods used for the open-loop constraint.

Results are given in Figure 14, in terms of a trade-off between the maximum allowable performance index (control cost) $\mathrm{J}$, and the minimum available structural mass. For this case, the open-loop flutter margin constraint is set at $-20 \%$ : the wing is open-loop unstable within the flight envelope, and must rely on the controller for both stability and the requisite $15 \%$ margin. For a control cost constraint limit set to a higher value (above 100 in Figure 
14), the optimizer can easily satisfy both the cost constraint and the closed-loop flutter constraint without adding any additional mass to the structure. The optimal mass in this extreme case $(13,058 \mathrm{~kg})$ is the same as the wing mass in the previous section (Figure 11), when the required open-loop margin is set to $-20 \%$, and no controller is utilized. In other words, the mass penalty paid to obtain a flutter-free structure in the previous section is entirely negated with the LQR controller.

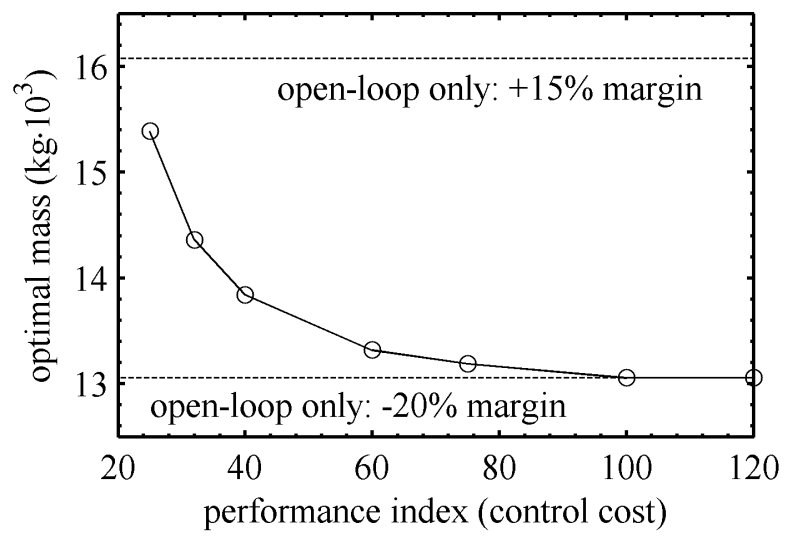

Figure 14. Trade-off between the allowable LQR control cost and the minimum structural mass.

Lower values (below 100 in Figure 14) of the performance index limit present a harder set of constraints to atisfy, and the LQR controller is unable to stabilize the system at a $15 \%$ margin without exceeding the allowable cost. The optimizer is then forced to add additional structure to the wing (i.e., stiffen the wing) to help satisfy the closed-loop flutter margin. A mass penalty of $17.7 \%$ is paid when the allowable control cost is decreased from 100 to 25. Though not shown, the optimizer also re-adjusts the quasi-steady control surface rotations, $\boldsymbol{\theta}_{\text {in }}$. These design variables have no explicit effect on either the wing mass objective or the flutter constraints, but can help maintain static aeroelastic constraints when material is re-allocated to satisfy dynamic constraints.

Very low allowable values of $J$ in Figure 14 (below 25) will eventually force the optimizer to stiffen the wing to a point where the resulting structure weighs more than if no controller had been utilized at all, with the open-loop margin set to $15 \%$. This mass (marked in the figure) is $16,075 \mathrm{~kg}$, which is also the heaviest data point in Figure 11 . The optimizer is unable to recognize this contradiction, as it is forced to satisfy control cost and closed-loop margin constraints, but the controller should, in reality, be disconnected in these situations.

The control weighting design variables $\boldsymbol{R}$ in Figure 14 all largely reside at the lower bound, which is set to 50 . As the allowable value of $J$ is decreased (i.e., as the constraint becomes harder to satisfy), the optimizer moves the LQR design speed, $U_{L Q R}$, down. As noted above, values of $U_{L Q R}$ below the open-loop flutter speed can decrease the control cost (as the instabilities at $U_{L Q R}$ are less aggressive), but this also renders the controller less effective. This effectiveness decreases to a point where the optimizer must eventually stiffen the wing to help meet the closed-loop flutter margin, as seen in Figure 14.

The case shown in Figure 14, where the open-loop margin is set to $-20 \%$, is an extreme case in the sense that the controller is forced to stabilize a structure with aggressive instabilities within the flight envelope. It is expected that increasing the open-loop flutter margin to higher values will alleviate the controller's burden, and decrease the mass penalty paid for a given control cost constraint limit. As in the quasi-steady maneuver load examples (Figure 9), increasing the open-loop margin to the same $15 \%$ value used for the closed-loop margin is the limiting case, at which point the role of the LQR controller during the optimization process is completely removed.

Open- and closed-loop eigenvalue migration plots (real part) are shown in Figure 15, for the case where the performance index constraint is set to 100 . It can be seen that both the open-loop constraint (-20\% margin) and the closed-loop constraint $(+15 \%$ margin) are highly active. The closed-loop case, in particular, has two modes closely interacting with the constraint boundary. The closed-loop case also demonstrates one unstable wind-off mode. Similar behavior is noted in Refs. [4] and [35], and is presumably due to the stabilizing aerodynamic forces utilized by the controller at $U_{L Q R}$, but which are not present at very low dynamic pressures. A long-term solution to this problem is gain scheduling [33], which is not utilized here, or simply disconnecting the controller at lower dynamic pressures. As the mode re-stabilizes for higher speeds (as guaranteed by the LQR methodology), the mode is simply ignored for the purposes of computing the flutter constraint in Eq. 6. 

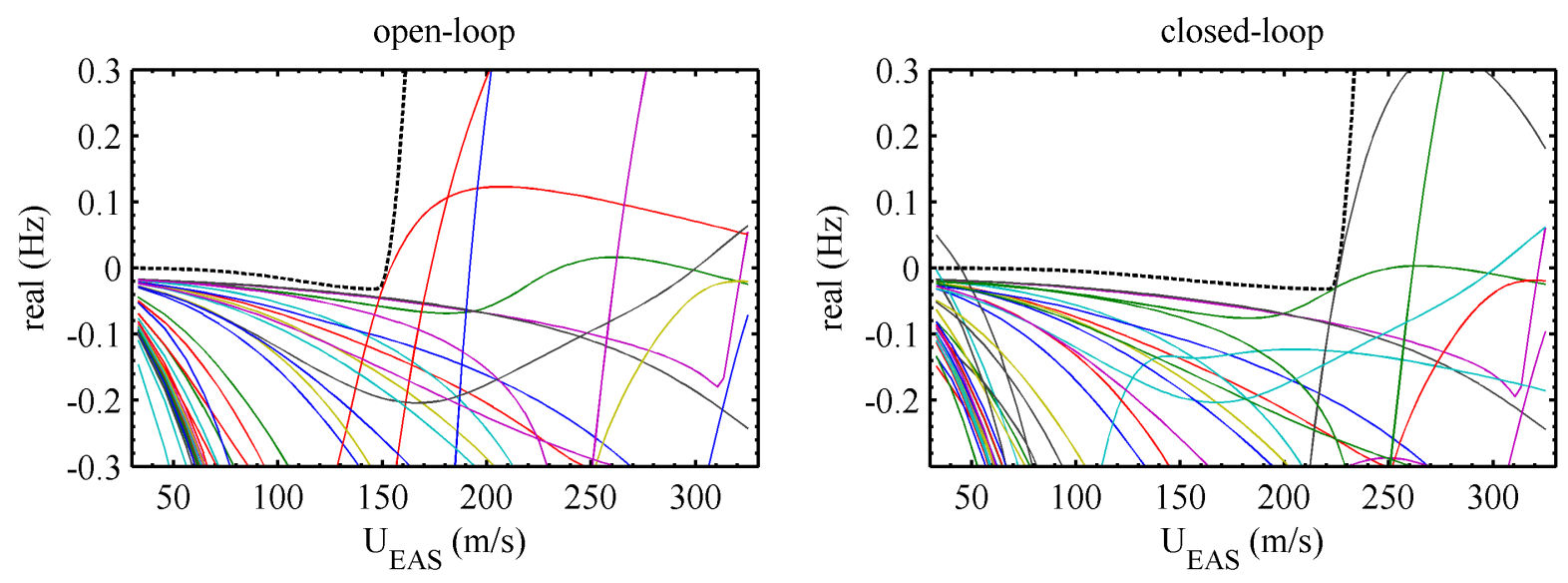

Figure 15. Open- (left) and closed-loop (right) real-part eigenvalues under active flutter constraints.

The closed-loop flutter mode shapes (eigenvectors of the flutter eigenvalue, at the flutter speed) are shown in Figure 16 and Figure 17 for the wing deformation and the corresponding VCCTEF inputs $\left(\boldsymbol{u}_{A S E}\right)$, respectively. Wing deformation is primarily a first-bending shape with an out-of-phase torsional component indicated by the imaginary part. As noted above, VCCTEF inputs are only present for the last row of control surface segments, actuated with electric drive motors. These input actuation rotations are largest towards the wing tip, where they generate negative lift in order to counteract the large positive bending deformation of the wing.

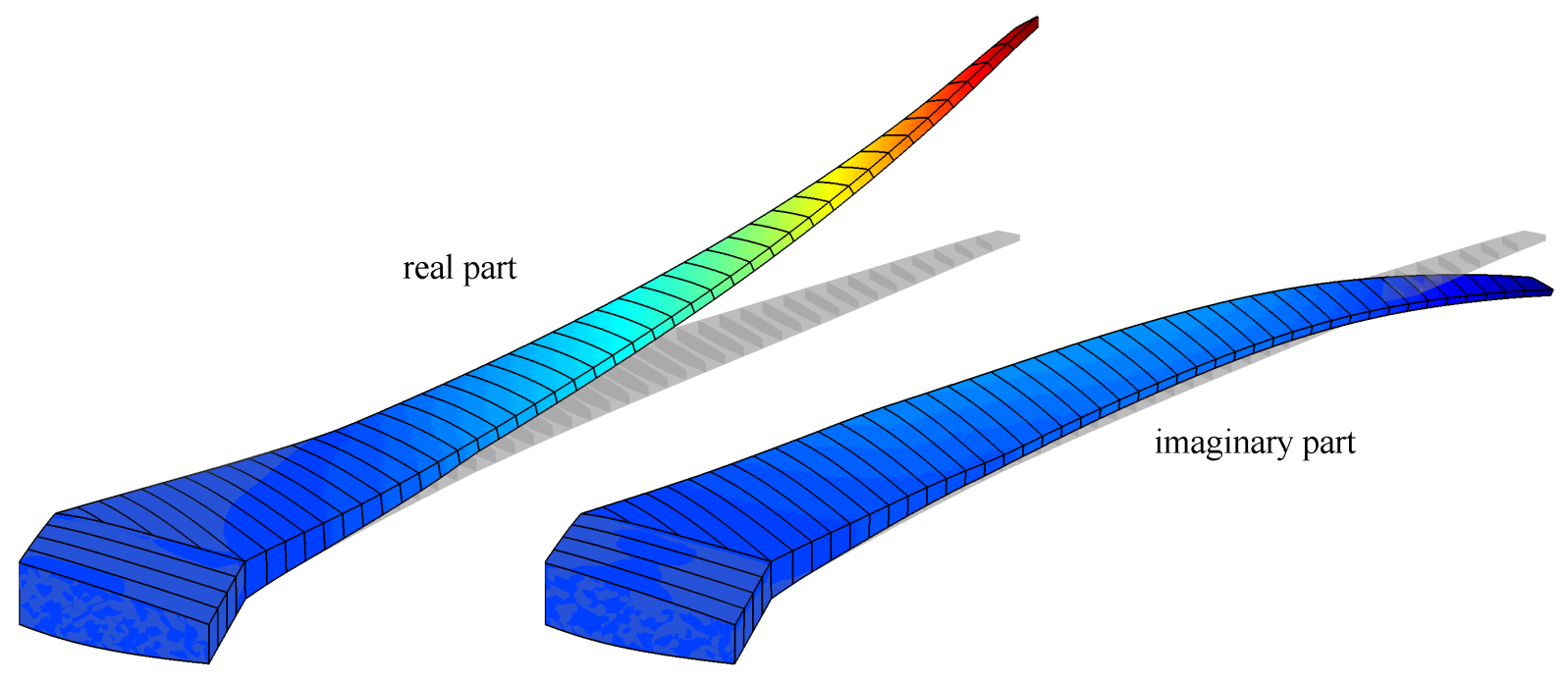

Figure 16. Closed-loop flutter mode shapes.

VI. Conclusions

This paper has conducted aeroservoelastic optimization of the Common Research Model (uCRM) outfitted with Variable Camber Continuous Trailing Edge Flaps: VCCTEF. This actuation concept involves several spanwise flaps along the trailing-edge of the wing, with each flap broken into three chordwise segments. The first two segments are actuated with slow-response shape memory allow torque-tube actuators, while the third is actuated with much faster (but with poorer hinge moment characteristics) electric drive motors. Each spanwise flap is connected to adjacent flaps with an elastomeric skin, to preserve a continuously deforming trailing-edge shape. This variable camber actuation system is used in two ways: quasi-steady maneuver load alleviation, and unsteady active flutter suppression.

The objective of the optimization procedure is to minimize the structural weight of the wingbox. Design variables include shell thickness, stiffener thickness, and stiffener height of numerous rib, spar, and skin design patches, quasi-steady actuation deflections of the VCCTEF system, and LQR controller design parameters. Design 
constraints include maneuver stresses and skin buckling, hinge moments, elastomer skin strains, stiffener geometry restrictions, open-loop flutter margin, closed-loop flutter margin, and LQR control cost.

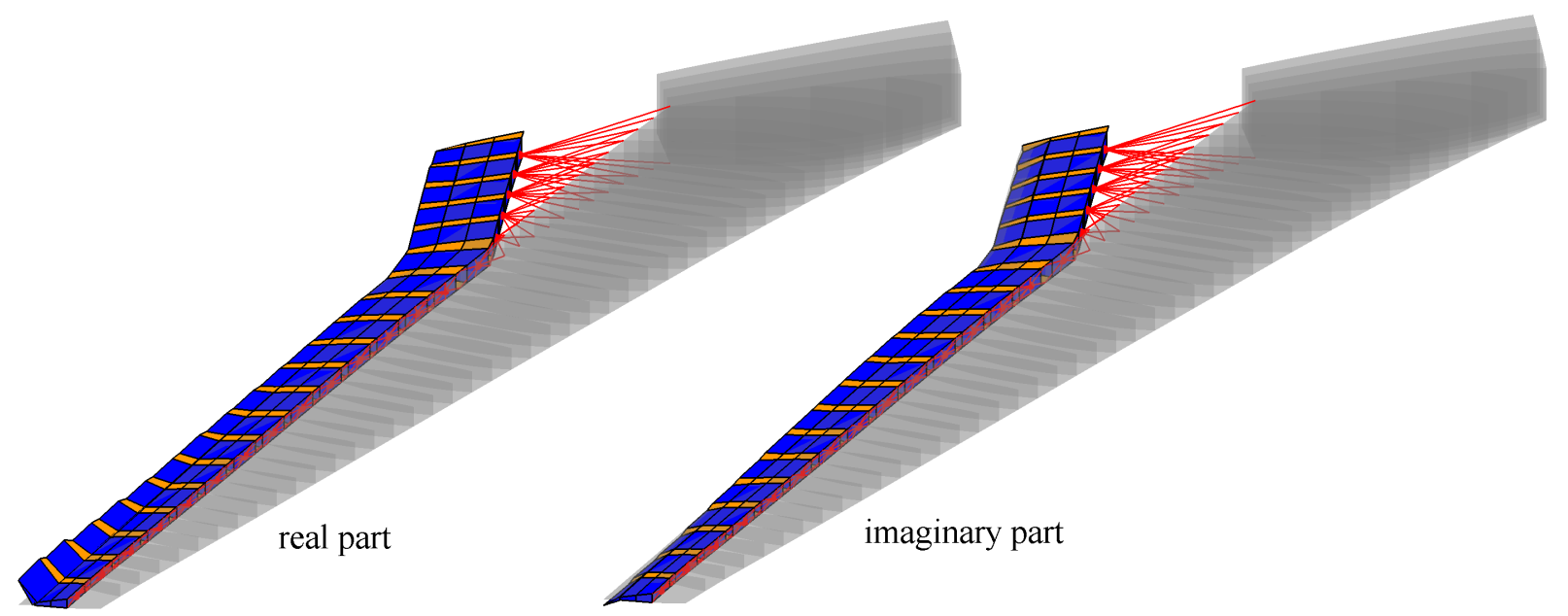

Figure 17. Oscillatory input VCCTEF deflections corresponding to the mode shapes of Figure 16.

In general, the VCCTEF system was found capable of substantial aeroelastically-feasible reductions in structural weight. Two important caveats to this success are explored here, however. First, open-loop load cases (where the VCCTEF system is not active) could be considered in the optimization process, even though they are not expected to be encountered in normal flight operations. Using low values for the safety factor (steady cases) or flutter margin (unsteady cases) of these open-loop cases devalues their role relative to the closed-loop cases (where the VCCTEF system is active), and allows for large mass reductions, albeit with higher risk. The opposite is true for large values of safety factor and/or flutter margin. A second caveat is the cost of operating the VCCTEF system: constraints placed on these cost metrics limit their ability to beneficially alter the aeroelastic behavior of the wing, and therefore limit the mass reductions available through optimization.

\section{Acknowledgements}

This work is funded by NASA's Advanced Air Transport Technologies program. Thanks to Dr. Joaquim Martins and Dr. Gaetan Kenway of the University of Michigan for providing the uCRM model.

[1] Livne, E., "Integrated Aeroservoelastic Optimization: Status and Direction," Journal of Aircraft, Vol. 36, No. 1, pp. 122-145, 1999.

[2] Zink, P., Mavris, D., Raveh, D., "Maneuver Trim Optimization Techniques for Active Aeroelastic Wings," Journal of Aircraft, Vol. 38, No. 6, pp. 1139-1146, 2001.

[3] Raveh, D., "Maneuver Load Analysis of Overdetermined Trim Systems," Journal of Aircraft, Vol. 45, No. 1, pp. 119-129, 2008.

[4] Zeiler, T., Weisshaar, T., "Integrated Aeroservoelastic Tailoring of Lifting Surfaces," Journal of Aircraft, Vol. 25, No. 1, pp. 76-83, 1988.

[5] Dracopoulos, T., Oz, H., "Integrated Aeroelastic Control Optimization of Laminated Composite Lifting Surfaces," Journal of Aircraft, Vol. 29, No. 2, pp. 280-288, 1992.

[6] Nam, C., Kim, Y., "Optimal Design of Composite Lifting Surface for Flutter Suppression with Piezoelectric Actuators," AIAA Journal, Vol. 33, No. 10, pp. 1897-1904, 1995.

[7] Jackson, T., Livne, E., "Integrated Aeroservoelastic Design Optimization of Actively Controlled StrainActuated Flight Vehicles," AIAA Journal. Vol. 52, No. 6, pp. 1105-1123, 2014.

[8] Pettit, C., "Uncertainty Quantification in Aeroelasticity: Recent Results and Research Challenges," Journal of Aircraft, Vol. 41, No. 5, pp. 1217-1229, 2004.

[9] Livne, E., Schmidt, A., Friedmann, P., "Integrated Structure/Control/Aerodynamic Synthesis of Actively Controlled Composite Wings," Journal of Aircraft, Vol. 30, pp. 387-394, 1993.

[10] Bindolino, G., Ricci, S., Mantegazza, P., "Integrated Servostructural Optimization in the Design of Aerospace Systems, Journal of Aircraft, Vol. 36, pp. 167-175, 1999. 
[11] Nguyen, N., Lebofsky, S., Ting, E., Kaul, U., Chaparro, D., Urnes, J., "Development of Variable Camber Continuous Trailing Edge Flap Performance Adaptive Aeroelastic Wing," SAE Technical Paper 2015-01-2565, 2015.

[12] Kenway, G., Martins, J., Kennedy, G., "Aerostructural Optimization of the Common Research Model Configuration," AIAA Aviation Conference, Atlanta, GA, June 16-20, 2014.

[13] Vassberg, J., DeHaan, M., Rivers, S., Wahls, R., "Development of a Common Research Model for Applied CFD Validation Studies," AIAA Applied Aerodynamics Conference, Honolulu, Hawaii, August 10-13, 2008.

[14] Stroud, W., Agranoff, N., "Minimum-Mass Design of Filamentary Composite Panels under Combined Loads: Design Procedure Based on Simplified Buckling Equations," NASA TN D-8257, 1976.

[15] Ting, E., Dao, T., Nguyen, N., "Aerodynamic Load Analysis of a Variable Camber Continuous Trailing Edge Flap System on a Flexible Wing Aircraft," AIAA SciTech Conference, Kissimmee, FL, January 5-9, 2015.

[16] Cook, R., Malkus, D., Plesha, M., Witt, R., Concepts and Applications of Finite Element Analysis, Wiley, New York, 2002.

[17] Katz, J., Plotkin, A., Low-Speed Aerodynamics, Cambridge University Press, Cambridge, UK, 2001.

[18] Appa, K., "Finite-Surface Spline," Journal of Aircraft, Vol. 26, No. 5, pp. 495-496, 1989.

[19] Kreisselmeier, G., Steinhauser, R., "Systematic Control Design by Optimizing a Vector Performance Index", International Federation of Active Controls Symposium on Computer-Aided Design of Control Systems, Zurich, Switzerland, 1979.

[20] Herencia, J., Weaver, P., Friswell, M., "Morphing Wing Design via Aeroelastic Tailoring," AIAA Structures, Structural Dynamics, and Materials Conference, Honolulu, HI, April 23-26, 2007.

[21] Dillinger, J., Klimmek, T., Abdalla, M., Gürdal, Z., "Stiffness Optimization of Composite Wings with Aeroelastic Constraints," Journal of Aircraft, Vol. 50, No. 4, pp. 1159-1168, 2013.

[22] Adelman, H, Haftka, R., "Sensitivity Analysis of Discrete Structural Systems," AIAA Journal, Vol. 24, No. 5, pp. 823-832, 1986.

[23] “777-200/300 Airplane Characteristics for Airport Planning," Boeing Commercial Airplanes, Report D6-58329, Renton, WA, 1998.

[24] Sigmund, O., "Morphology-Based Black and White Filters for Topology Optimization," Structural and Multidisciplinary Optimization, Vol. 33, pp. 401-424, 2007.

[25] Svanberg, K., "A Class of Globally Convergent Optimization Methods Based on Conservative Convex Separable Approximations," SIAM Journal of Optimization, Vol. 12, pp. 555-573, 2002.

[26] Kline, K., "Dynamic Analysis Using a Reduced Basis of Exact Modes and Ritz Vectors," AIAA Journal, Vol. 24, No. 12, pp. 2022-2029, 1986.

[27] Roger, K., "Airplane Math Modeling Methods for Active Control Design," AGARD CP-228, pp. 4-11, 1977.

[28] Hajela, P., "A Root Locus-Based Flutter Synthesis Procedure," Journal of Aircraft, Vol. 20, pp. 1021-1027, 1983.

[29] Ringertz, U., “On Structural Optimization with Aeroelasticity Constraints," Structural Optimization, Vol. 8, pp. 16-23, 1994.

[30] Dodd, A., Kadrinka, K., Loikkanen, M., Rommel, B., Sikes, G., Strong, R., Tzong, T., “Aeroelastic Design Optimization Program," Journal of Aircraft, Vol. 27 No. 12, pp. 1028-1036, 1990.

[31]Zhang, Z., Yang, S., Chen, P., "Linearized Euler Solver for Rapid Frequency-Domain Aeroelastic Analysis," Journal of Aircraft, Vol. 49, No. 3, pp. 922-932, 2012.

[32] Stevens, B., Lewis, F., Aircraft Control and Simulation, John Wiley and Sons, Hoboken, NJ, 2003.

[33] Barker, J., Balas, G., "Comparing Linear Parameter-Varying Gain-Scheduled Control Techniques for Active Flutter Suppression," Journal of Guidance, Control, and Dynamics, Vol. 23, No. 5, pp. 948-955, 2000.

[34] Gilbert, M., "An Analytical Sensitivity Method for use in Integrated Aeroservoelastic Aircraft Design," Mechanical Systems and Signal Processing, Vol. 4, No. 3, pp. 215-231, 1990.

[35] Moulin, B., "Modeling of Aeroservoelastic Systems with Structural and Aerodynamic Variations," AIAA Journal, Vol. 43, No. 12, pp. 2503-2513, 2005. 